\title{
Citrullinated Histone H3 Level as a Novel Biomarker in Pediatric Clinical Sepsis
}

\author{
Ronald Chandra, Antonius Hocky Pudjiadi*, Rismala Dewi \\ Department of Child Health, Cipto Mangunkusumo Hospital/Faculty of Medicine, Universitas Indonesia, Jl. Diponegoro No.71, Jakarta, Indonesia \\ *Corresponding author. E-mail: ahpudjiadi@gmail.com
}

Received date: Apr 23, 2021; Revised date: Aug 12, 2021; Accepted date: Aug 16, 2021

\section{Abstract}

$\mathrm{B}$ ACKGROUND: Sepsis is still leading cause of death in critically ill children. Early recognition of sepsis and treatments are needed to reduce its mortality. The use of citrullinated Histone H3 (Cit-H3) as an early sepsis marker and outcome predictor has been validated in previous studies among adults. However, only one study in pediatric meningococcal sepsis was reported with contradictory results. This study aims to determine Cit-H3 levels in pediatric clinical sepsis and analyze its association with sepsis severity and survival rate.

METHODS: A prospective observational cohort study involving 67 pediatric subjects clinically diagnosed with sepsis was conducted. Cit-H3 levels, Pediatric Logistic Organ Dysfunction-2 (PELOD-2) score, and Pediatric Sequential Organ Failure Assessment (pSOFA) score were assessed at the time of diagnosis (0-hour) and 48 hours later. Pearson Correlation test was used to determine the correlation between Cit-H3 levels with PELOD-2 and
pSOFA scores and receiver operating curve to find the cutoff of Cit-H3 levels on clinical sepsis patients.

RESULTS: Among clinically sepsis patients, the median Cit-H3 level was 1,210 (800-32,160) ng/mL, with optimal cut-off point $\geq 1200 \mathrm{ng} / \mathrm{mL}$ (sensitivity $83.3 \%$ and specificity $75.7 \%$ ) to discriminate sepsis. The median Cit-H3 levels at 0 -hour were lower in survivor compared to non-survivor group ( $p=0.016$ ). Cit-H3 level was able to predict mortality with optimal cut-off point $\geq 1,200 \mathrm{ng} / \mathrm{mL}$, sensitivity $72.2 \%$ and specificity $57.1 \%$ (AUC of $69.2 \%$; $p=0.017$ ). Using survival analysis, Cit-H3 was significantly associated with the mortality rate ( $p=0.023$; hazard ratio of 3.45).

CONCLUSION: Cit-H3 level could be potential to predict pediatric sepsis events and its outcome.

KEYWORDS: citrullinated histone H3, neutrophil extracellular traps, pediatric sepsis, PELOD-2 score, pSOFA score, survival

Indones Biomed J. 2021; 13(3): 316-23

\section{Introduction}

Sepsis still accounts for the highest morbidity and mortality in children. Despite advances and developments in monitoring, antibiotics, and mechanical ventilation support, sepsis-associated mortality remains high.(1) The reported incidence of pediatric severe sepsis in USA varies around $4.4-25.9 \%$ with a mortality rate of $8.2-40 \%$.(2)

Past definition of sepsis used systemic inflammatory response syndrome (SIRS) caused by infection, however, this was criticized as being too general.(3) In 2016, SEPSIS-3 released a new definition of sepsis, which is additional organ dysfunction due to dysregulated immune responses against infection.(4) Such criteria have yet to be validated for the pediatric population. In children, organ dysfunction and sepsis severity are often reported using pediatric logistic organ dysfunction (PELOD)-2 score and Pediatric Sequential Organ Failure Assessment (pSOFA) score.(5-6) However, diagnosis of sepsis using these criteria to determine organ dysfunction often results in under-and late diagnosis.

There is a need for an objective marker to guide therapy decisions and ultimately improve patient's survival. (7) Clinicians have been trying to pinpoint a marker of dysregulated immune response to predict organ dysfunction 
earlier, as symptoms of organ dysfunction usually appear late.(8-9) Therefore, a robust sepsis marker is needed to support clinical features in identifying infection cases, evaluate organ dysfunction severity, monitor therapeutic response, predict sepsis outcomes and/or limit unnecessary examination and management.(10)

In sepsis pathophysiology, pathogen interaction with pattern recognition receptors (PRRs) expressed in nonspecific immune cells activates nuclear factor- $\kappa \beta$ (NF$\kappa \beta)$ and releases pro-inflammatory cytokines. Subsequently, neutrophils are recruited infection site to prevent spreading into the circulation.(11) The key to successful pathogen clearance lies in neutrophil recruitment efficiency and its antimicrobial activity.

Neutrophils eliminate pathogens via phagocytosis, degranulation, and the formation of neutrophil extracellular traps (NETs).(12) The formation of NETs is an actively controlled process. It consists of a nucleus (DNA chromatin and histone) and granular neutrophils. However, NETs can act as a double-edged sword. It provides defense by ensnaring and killing pathogens, on the other side causing tissue damage, inflammatory cell activation, contributing to pathological SIRS, sepsis, and many pathological conditions.(13-14)

Previous studies reported the role of NETs on sepsis as an early sepsis marker (15-17), predictor of organ dysfunction $(14,18)$, thromboembolism (14), sepsis outcome (14), and as a response and target therapy (15). Surrogates of NETs for instance, cell-free DNA (cf-DNA), nucleosome, NETs related to neutrophil elastase enzyme (NE) and myeloperoxidase (MPO), and citrullinated histone H3 (Cit-H3). Among these, Cit-H3 assessment using enzyme-linked immunosorbent assay (ELISA) has been validated and deemed reliable as a surrogate marker of NETs.(19)

Many evidence reported superiority of NETs role as an early sepsis marker, organ dysfunction and sepsis outcome predictor, and therapeutic response indicator. However, the study on NETs in the pediatric population is still limited. There is only one report in pediatric meningococcal sepsis that uses the MPO-DNA complex as a NETs marker. However, their findings reported a poor correlation to organ dysfunction and outcome, contrary to the results on septic animal and adult studies.(20) Because of these contradictory results, the role of NETs by using Cit-H3 as a surrogate marker in children with clinical sepsis need to be investigated further. The aim of this study is to determine the plasma levels of Cit-H3 in children with clinical sepsis and evaluate its relationship to severity and survival.

\section{Methods}

A prospective cohort study was conducted at Cipto Mangunkusumo Hospital, Jakarta from February until May 2018. Ethical approval was obtained from Faculty of Medicine Universitas Indonesia Institutional Review Board (Approval number: 0046/UN2.F1/ETIK/2018).

\section{Study Population}

Inclusion criteria were children aged 1 month to 18 years old with clinical sepsis diagnosed at emergency room (ER) or pediatric intensive care unit (PICU) and hospitalized for 7 days or more. Subjects with autoimmune, malignancy, trauma, and post-operative subjects were excluded. The sample size for this study is 66 patients estimated by using the formula for pilot study.(17) Consecutive sampling was performed.

\section{Diagnostic Criteria}

Onto subjects who fulfilled inclusion criteria, necessary examination for sepsis severity using the PELOD-2 and pSOFA scores along with $\mathrm{Cit}-\mathrm{H} 3$ examination was performed.

\section{PELOD-2 Score}

PELOD-2 score consists of 10 variables which represent 5 organ dysfunctions, which includes Glasgow coma score, pupillary reaction, lactatemia, mean arterial pressure, serum creatinine, partial pressure of oxygen, partial pressure of carbon dioxide, the use of mechanical ventilation, white blood cell count, and platelet counts. PELOD-2 score assesses the severity of multiple organ dysfunction syndrome. A higher PELOD-2 score correlates with a higher number of organ failures and mortality rate. The cut-off of 10 in PELOD-2 score was used to determine life-threatening organ dysfunction.

\section{pSOFA Score}

pSOFA score assesses 6 organ systems such as respiratory, hematological, hepatic, cardiovascular, neurological, and renal systems every 24-hour period. Daily pSOFA score was the sum of the 6 sub-scores. A higher score implies a worse prognosis.

\section{Cit-H3 Examination}

A total of $3 \mathrm{~mL}$ of venous blood in EDTA was obtained for Cit-H3 examination. Immediately, centrifugation was performed at 2,000 RPM for 20 minutes. The plasma 
sample was separated and stored at $-70^{\circ} \mathrm{C}$. Examination was performed simultaneously after all samples had been collected. EpiQuikTM Circulating Histone H3 Citrullination was carried out at Prodia Laboratory, Jakarta. The examination was carried out at 0 -hour (at the time of diagnosis) and 48-hour following sepsis diagnosis.

Based on the SEPSIS-3 definition, subjects were divided into suspected sepsis and sepsis groups. The suspected sepsis group is the patient with clinical sepsis with PELOD score $<10$. Sepsis diagnosis was based on suspected or confirmed infection and life-threatening organ dysfunction. Infection is suspected based on physical examination and laboratory findings related to the focus of infection (such as dysuria, respiratory disorder with abnormal lung auscultation, meningitis sign, purulent wound, rash) (21), accompanied by systemic inflammatory response syndrome (SIRS) (22). Organ dysfunctions was defined as any clinical findings of neurological, cardiovascular, respiratory, hematological, hepatic and/or renal system dysfunction.(23) Early mortality was defined as mortality within 7-days since the diagnosis of clinical sepsis.

\section{Data Collection and Processing}

The baseline characteristics of all 67 patients were collected from the medical record. The data collected were age, gender, duration of illness, nutritional status, focus of infection (classified by clinical diagnosis), positive culture, leukocyte count, thrombocyte count, procalcitonin, PELOD-2 score, pSOFA score, medications, and seven days mortality.

\section{Statistical Analysis}

Statistical analysis was performed on SPSS version 20.0 (IBM Corporation, Armonk, NY, USA) with $p$-value $<0.05$ considered as significant. Correlation between Cit-H3 and severity of the disease (measured by PELOD-2 and pSOFA scoring) was conducted using Spearman's rho at hour-0 and Pearson at h-48. Pooled analysis was assessed using Pearson.

\section{Results}

\section{Baseline Characteristics}

Sixty-seven patients were eligible to be included in this study. Thirty-seven $(55.2 \%)$ patients were suspected sepsis, $15(22.3 \%)$ patients had sepsis and 15 (22.3\%) patients had septic shock at the time of diagnosis. Eighteen (27\%) patients died within seven days of follow up, eight of them died before 48 hours. Thus, fifty-nine patients were included in the 48-hours measurement of Cit-H3 levels. The overall hospital mortality rate was $37.3 \%$. The baseline characteristics of sepsis patients were shown in Table 1. There were 37 children with suspected sepsis and 30 children with sepsis at the beginning of sampling.

\section{Cit-H3 Level in Serum}

Median 0-hour Cit-H3 level in suspected sepsis group was $1,080(800-12,110) \mathrm{ng} / \mathrm{mL}$, significantly lower compared to sepsis group of $2,110(860-32,160) \mathrm{ng} / \mathrm{mL}(p<0.001)$. The discrimination value of Cit-H3 levels as a predictor of sepsis in clinical sepsis patients was 77.2\% (95\% CI: $64.6-89.7 \% ; p<0.001)$. The Youden Index was 0.59 . The optimal cut-off point of 0 -hour Cit-H3 level in predicting sepsis was $1,200 \mathrm{ng} / \mathrm{mL}$ with $83.3 \%$ sensitivity and $75.7 \%$ specificity. Using Cit-H3 level $\geq 1200 \mathrm{ng} / \mathrm{mL}$ as sepsis marker, a positive likelihood ratio ( $\mathrm{LR}+$ ) of 3.4 (1.9-6.8) and a negative likelihood ratio (LR-) of 0.22 (0.1-0.5) were obtained. The pretest probability is 0.44 . The ROC curve is shown in Figure 1.

\section{Association between Cit-H3 and Disease Severity}

From 126 samples obtained at 0-hour $(n=67)$ and 48-hour $(\mathrm{n}=59)$, Cit-H3 was poorly correlated with PELOD-2 $(\mathrm{r}=0.377 ; p<0.001)$ and pSOFA score $(\mathrm{r}=0.243 ; p=0.006)$. Subgroup analysis shows that 0 -hour and 48-hour Cit-H3 levels were moderately correlated with PELOD-2 score. Cit-H3 levels demonstrate a weak correlation with pSOFA score at 0 -hour, but not at 48-hour. Correlation between Cit-H3 levels and sepsis severity is summarized in Table 2. The progression of the disease using PELOD-2 score was measured at 48-hour. Changes in the Cit-H3 levels (delta Cit-H3) among clinical sepsis group were significantly different between subjects whom clinical conditions worsened, unchanged, and improved within 48 hours (40 (-370-4,510) vs. $15(-10,095-5,380)$ vs. -195 (-6,010-6,450) $\mathrm{ng} / \mathrm{mL}$ respectively, $p=0.046)$.

\section{Cit-H3 and Mortality}

Survivors in the clinical sepsis group had a significantly lower median 0-hour Cit-H3 level compared to nonsurvivors $(p=0.016)$. However, no difference in hour-48 Cit-H3 and delta Cit-H3 levels were found between the survivor and non-survivor, as shown in Table 3.

The area under the curve (AUC) for 0-hour Cit-H3 as a mortality predictor in clinical sepsis patients was $69.2 \%$ (95\% CI: 55-83.4\%; $p=0.017)$. The Youden Index was 0.293. The optimal cut-off point of hour-0 Cit-H3 level to 
Table 1. Baseline characteristics of clinical sepsis patients.

\begin{tabular}{|c|c|}
\hline Characteristics & $\begin{array}{l}\text { Value } \\
(n=67)\end{array}$ \\
\hline Age, median (min-max) & $14(1-212)$ \\
\hline \multicolumn{2}{|l|}{ Gender, n $(\%)$} \\
\hline Male & $41(61)$ \\
\hline Female & $26(39)$ \\
\hline Duration of illness (days), median (min-max) & $7(1-92)$ \\
\hline \multicolumn{2}{|l|}{ Nutritional status, n (\%) } \\
\hline Obesity & $4(6)$ \\
\hline Normal nutritional status & $23(34)$ \\
\hline Wasted & $15(23)$ \\
\hline Severely wasted & $25(37)$ \\
\hline \multicolumn{2}{|l|}{ Infection source, $\mathrm{n}(\%)$} \\
\hline Neurological & $5(8)$ \\
\hline Respiratory & $54(81)$ \\
\hline Abdomen & $7(10)$ \\
\hline Other & $1(2)$ \\
\hline Positive culture, $\mathrm{n}(\%)$ & $48(72)$ \\
\hline Leukocyte, $/ \mathrm{mm}^{3}$, median (min-max) & $15,000(1,120-72,490)$ \\
\hline Thrombocyte, $/ \mathrm{mm}^{3}$, mean \pm SD & $238,989.40 \pm 164,490.64$ \\
\hline Procalcitonin, $\mathrm{ng} / \mathrm{mL}$, median (min-max) & $7.02(0.02-368)$ \\
\hline Cit-H3 plasma concentration, ng/mL, median (min-max) & $1,210(800-32,160)$ \\
\hline PELOD-2 score, median (min-max) & $9(0-18)$ \\
\hline pSOFA score, median (min-max) & $9(4-17)$ \\
\hline \multicolumn{2}{|l|}{ History use of } \\
\hline Inotropic/vasoactive, yes, n (\%) & $24(36)$ \\
\hline Mechanical ventilation, yes, $\mathrm{n}(\%)$ & $49(73)$ \\
\hline Steroid, yes, $\mathrm{n}(\%)$ & $3(4)$ \\
\hline Seven days mortality, n (\%) & $18(27)$ \\
\hline
\end{tabular}

predict early mortality in clinical sepsis patients was 1,155 $\mathrm{ng} / \mathrm{mL}$ (77.8\% sensitivity, $57.1 \%$ specificity). However, for clinical consideration, we used the cut-off point for hour0 Cit-H3 of $1,200 \mathrm{ng} / \mathrm{mL}$ as a predictor of early mortality in clinical sepsis $(72.2 \%$ sensitivity, $57.1 \%$ specificity, $\mathrm{LR}+=1.68, \mathrm{LR}-=0.49)$. The ROC curve is shown in Figure 1. Median survival length in clinical sepsis group 0-hour Cit-H3 $\geq 1,200 \mathrm{ng} / \mathrm{mL}$ was 5.21 days, shorter than group 0-hour Cit-H3 $<1200 \mathrm{ng} / \mathrm{mL}$ which were 6.72 days, $p=0.023)$. The hazard ratio of hour- 0 Cit-H3 level was 3.45 (95\% CI: 1.19-10.04). The Kaplan-Meier plot is shown in Figure 2.

\section{Discussion}

In this study, an association between NETs and disease severity and its use in prognostic survival predictor in pediatric clinical sepsis was demonstrated. Kinetic change of Cit-H3 level in 48 hours was also associated with disease progression (worsening, unchanged and improving). In clinical sepsis group, hour-0 Cit-H3 level was higher in nonsurvivor group than survivor group, but not in the 48-hour Cit-H3. In pediatric clinical sepsis, Cit-H3 level can be considered for both an early marker for sepsis and outcome predictor.

The level of Cit-H3 measured using ELISA method as NETs marker is reliable and has been validated on humans. (19-20) However, the use of Cit-H3 as a marker of NETs on sepsis is still limited to animal and on human models. On human model using LPS-induced inflammation study, healthy adults Cit-H3 level was reported at the lowest detection value of $5 \mathrm{ng} / \mathrm{mL}$. This increased within 3-4 hours LPS stimulation (28.7-93.2 ng/mL).(17) Previous study among adults reported Cit-H3 was significantly higher in septic shock patients (SP) compared to both healthy controls (HV) and non-infectious shock (NIC) at 0-hour [median (IQR): SP 101.5 pg/mL (67-166); HV 8 pg/mL 
A.

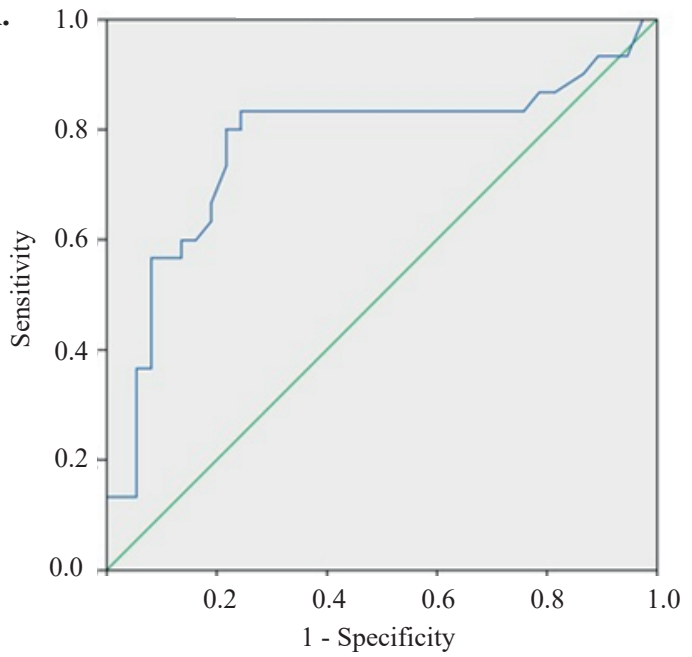

B.

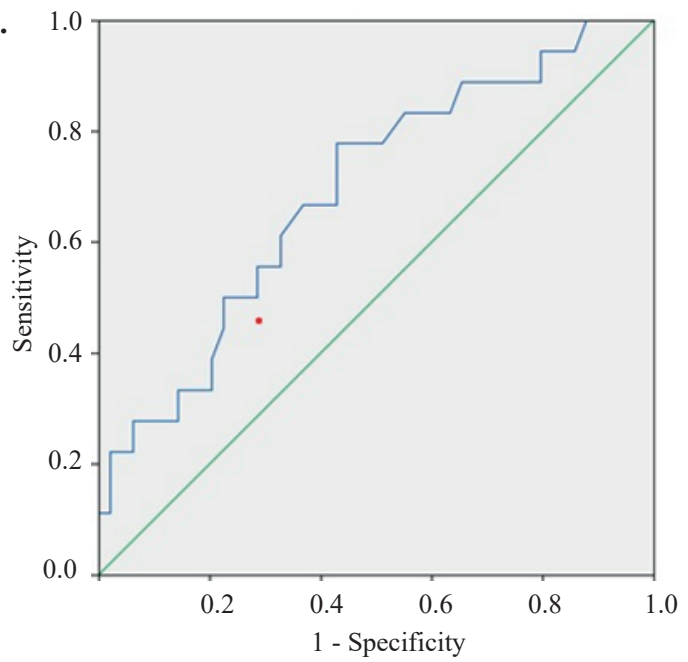

Figure 1. Receiver operating characteristics curve of Cit-H3 levels at 0-hours. A: as a sepsis predictor in children; B: as a mortality predictor in children

(0-30), $p<0.0001$; NIC $34 \mathrm{pg} / \mathrm{mL}(0-91.5), p<0.0001]$ and there was no significant difference between HV and NIC. (24) Sepsis in adults and pediatric both showed significantly higher Cit-H3 level than health controls.

In endotoxemia condition, neutrophils will migrate and bind endothelial cells of organ which is receiving a significant portion of cardiac output and portal vein drainage (lung and hepatic sinusoids).(25) After a while, the activated platelets will anchor to the surface of neutrophils in order to release NETs. In this study, a different of Cit-H3 levels statistically based on source of infections were not found. However, the highest Cit-H3 levels was found in the source of infection originating from abdomen, followed by respiration and CNS.

The platelets function as a barometer in the blood was reported will becoming activated only during severe infection in order to stimulate neutrophils to release NETs. In an animal model sepsis, injection LPS in low dose (1 ng/ $\mathrm{mL}$ ) or maximal doses for induction neutrophils to release NET was reported insufficient to activate platelets to bind neutrophils in order to release NETs, but the formation of
NETs will only occur on high doses of LPS. $(25,26)$ An increase of NETs was positively corelated with the dose of LPS.(27) In this study we found that the Cit-H3 levels in sepsis group was higher statistically than suspected sepsis group.

Detection of Cit-H3 should be done in critical acute phase after the formation of NETs as written in previous reports in pediatric meningococcal sepsis and adult sepsis. $(20,28)$ This study similarly obtain Cit-H3 from the newly diagnosed patient in the acute or acute on chronic process, hence its increase was strongly related to the acute process.

Findings in mice models showed that Cit-H3 could be used as a marker for endotoxemia sepsis. Upon LPS administration, an increase in Cit-H3 was detected within 0.5 hours and remained for up to 24 hours. Compared to other sepsis markers (procalcitonin 6, IL-1 $\beta$ and IL-6), Cit-H3 is more specific as it is only increased in inflammation caused by infection, rapidly detected in the blood, and has a long half-life.(15) Moreover, a study in adult with burn injury showed the role of Cit-H3 as an early specific predictor for sepsis and severe sepsis.(17) Thus, we evaluate its ability

Table 2. Correlation between Cit-H3 levels with PELOD-2 and pSOFA score.

\begin{tabular}{|c|c|c|c|c|}
\hline \multirow{3}{*}{$\begin{array}{c}\text { Cit-H3 levels } \\
\text { (ng/mL) }\end{array}$} & \multicolumn{4}{|c|}{ Sepsis Severity } \\
\hline & \multicolumn{2}{|c|}{ PELOD-2 Score } & \multicolumn{2}{|c|}{ pSOFA Score } \\
\hline & $\mathbf{R}$ & $p$-value & $\mathbf{R}$ & $p$-value \\
\hline 0 -hour $(\mathrm{n}=67)$ & $0.441^{\mathrm{a}}$ & $<0.001$ & $0.297^{\mathrm{a}}$ & 0.015 \\
\hline 48 -hour $(\mathrm{n}=59)$ & $0.401^{\mathrm{b}}$ & 0.002 & $0.161^{\mathrm{a}}$ & 0.224 \\
\hline Total $(n=126)$ & $0.377^{\mathrm{b}}$ & $<0.001$ & $0.243^{\mathrm{a}}$ & 0.006 \\
\hline
\end{tabular}

aSpearman's rho correlation test, ${ }^{b}$ Pearson correlation test. 
Table 3. Cit-H3 levels at $\mathbf{0}$-hour, 48 -hour, and changes in 48 hours in clinical sepsis patients between survived and death groups.

\begin{tabular}{llccc}
\hline \multirow{2}{*}{ Clinical Sepsis } & \multicolumn{2}{c}{ Cit-H3 levels $(\mathbf{n g} / \mathbf{m L})$} & \multirow{2}{*}{$\boldsymbol{p}$-value } \\
\cline { 3 - 4 } & & Median & Range & \\
\hline 0-hour & Survive $(\mathrm{n}=49)$ & 1,100 & $(800-25,900)$ & 0.016 \\
& Death $(\mathrm{n}=18)$ & 1,775 & $(950-32,160)$ & \\
\hline \multirow{2}{*}{ 48-hour } & Survive $(\mathrm{n}=49)$ & 1,200 & $(660-17,840)$ & 0.505 \\
& Death $(\mathrm{n}=10)$ & 1,255 & $(580-31,900)$ & \\
\hline
\end{tabular}

Mann Whitney U test.

to predict incidence of sepsis in children. We found that Cit-H3 $>1,200 \mathrm{ng} / \mathrm{mL}$ was able to predict sepsis event in children.

We measured the severity of clinical sepsis using both PELOD-2 and pSOFA score as both have excellent discriminative value. Currently, the most widely used scoring system to assess organ dysfunction in children is PELOD-2 score. Unlike PELOD-2, pSOFA is relatively newer and yet to be validated. Children in sepsis group (PELOD-2 score >10.5) had higher Cit-H3 levels compared to suspected sepsis group, indicating both infection and organ dysfunction were more severe in septic group.

Our study found a weak correlation between Cit-H3 levels and PELOD-2 and pSOFA score. This may be due to the small number of sample (power post $\mathrm{Hoc}<80 \%$ ) and practical issues in assessing the scores. Some subjects received vasoactive or inotropic drugs, hence affecting mean arterial pressure for PELOD-2 measurement. Assessment of pSOFA scores were also affected due to majority (73\%) subjects who required mechanical ventilation had $\mathrm{PF}$ ratio of $>200$, which is not representative of the true oxygenation index value. Poor correlation between pSOFA and hour- 0 Cit-H3 level may also be affected by blood gas analysis performed post-intubation and preexisting chronic disease that affected bilirubin levels. In our study, correlation between 0-hour NETs and PSOFA score is weaker than that of adult sepsis $(\mathrm{r}=0.511 ; p=0.0028) .(16)$ This finding may have been attributed due to the difference in patient characteristics and NETs assessment method. On that study, cf-DNA is used to measure NETs, which recently found to be unreliable as they may originate from necrotic tissue and apoptotic cells other than NETs. $(13,18)$

The kinetic of delta Cit-H3 levels in 48 hours were significantly different between conditions worsened, unchanged, and improved clinical septic groups. Existing reports show that antibiotics such as azithromycin,

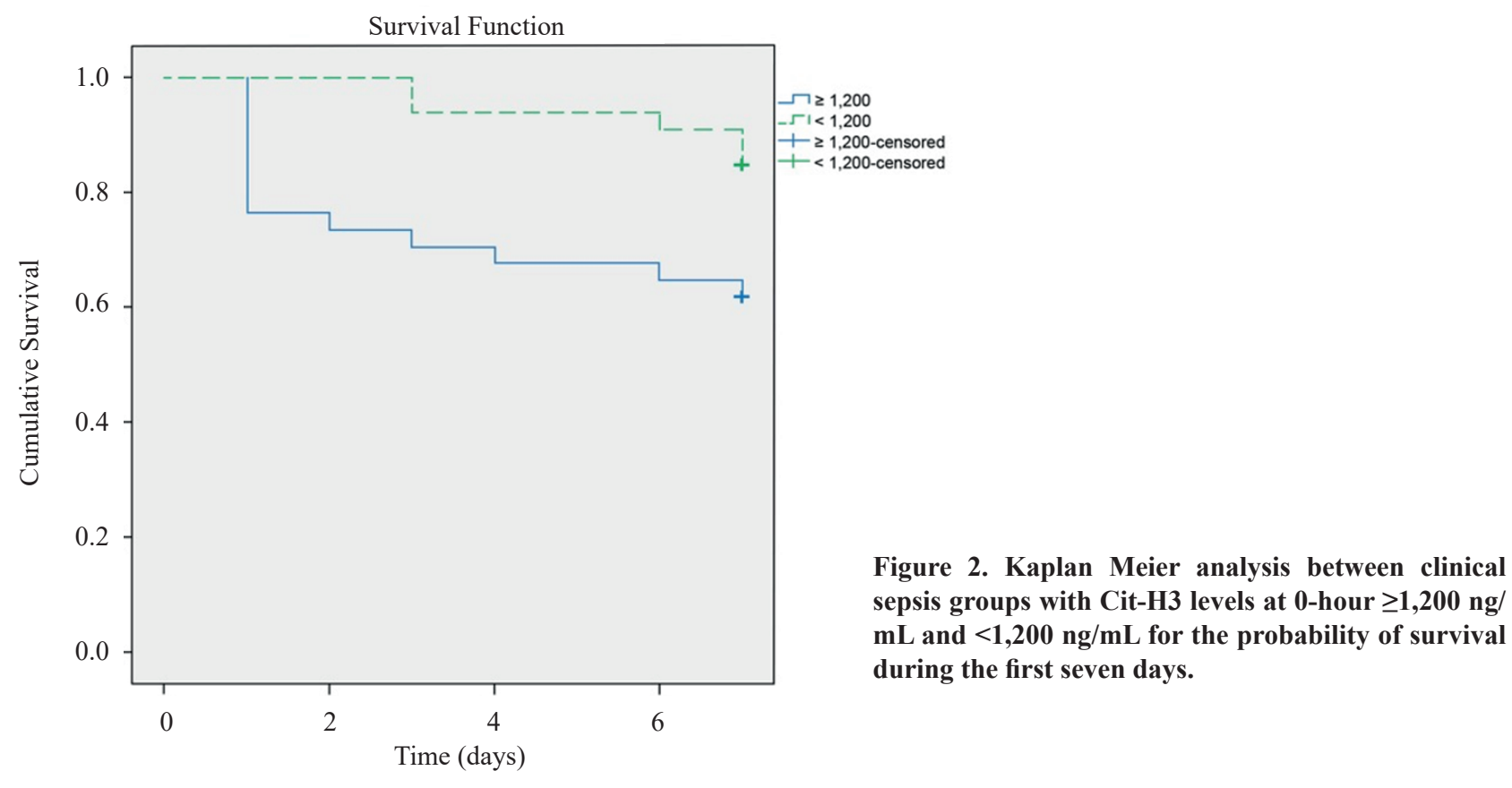


chloramphenicol (29), and gentamycin (30) inhibit NETs formation, while amoxicillin (31) and clarithromycin (32) induce NETs formation. In our study, only $16.6 \%$ subjects received antibiotics which have been shown to modulate NETs formation (lactams and macrolides). Most subjects received cefotaxime $(43.9 \%)$, which was shown to not affect NETs. NETs-modulating antibiotics used in our study may not significantly affect changes in Cit-H3 level, hence can still be used to monitor therapeutic response in clinical sepsis.

In this study, higher initial Cit-H3 levels were found in non-survivor compared to survivor of clinical sepsis group. Using the Cit-H3 cut-off of $1,200 \mathrm{ng} / \mathrm{mL}$, clinical sepsis patients with 0-hour Cit-H3 level $<1,200 \mathrm{ng} / \mathrm{mL}$ survived 3.45 times longer compared to 0 -hour Cit-H3 level $>1,200$ $\mathrm{ng} / \mathrm{mL}$. A study in children with meningococcal sepsis found that NETs level (on admission and 24-hour) was neither associated to disease severity nor outcome. No association established between NETs and sepsis severity in their study might be due to relatively small samples, difference in sepsis model and measuring instruments (NETs and disease severity assessment) and balanced charge between NETosis and cf-DNA affecting the quantification.(19-20)

The results of current study were similar to result of adult sepsis studies, in terms of significantly higher NETs level in non-survivors compared to survivors.(14) In experimental animals with septic shock, level of Cit-H3 is reported to be associated with LPS doses and mortality. The higher LPS doses correspond to higher Cit-H3 and earlier mortality. All mice which received higher LPS dose died within 23 hours, compared to only one died at 27 hours ( $p=0.00006)$ in those receiving lower LPS dose.(32) Another study in polymicrobial animal sepsis model also reported increase in NETs levels to be associated with 24hour mortality.(16) Although both reports were of animals studies, the relationship between NETs levels and sepsis mortality was shown to occur during the acute process.

This study has several limitations; a preliminary study required to be validated, heterogeneous subjects in the course of the disease and the severity of the disease; tertiary hospitals settings, no health controls included, small number of samples that yielded post Hoc power of less than $80 \%$. This study did not take into account the effect of therapeutic intervention and secondary infection after 48 hours which could affect 7-days mortality. Further study should be conducted, involving comparing with other inflammatory mediators and coagulation markers to evaluate the dynamics of NETs in the pathobiology process of organ dysfunction in pediatric sepsis.

\section{Conclusion}

The use of Cit-H3 as a novel biomarker in pediatric clinical sepsis presents a promising result. The findings from this study suggest that Cit-H3 level in pediatric clinical sepsis is significantly correlated to disease severity and survival rate.

\section{Acknowledgements}

We would like to acknowledge Ardiles Varian, M.D. and Angela Kimberly Tjahjadi, M.D. for proofreading and language editing of the manuscript for submission.

\section{Authors Contribution}

$\mathrm{RC}$ and AHP formulated the original research design and the writing of the paper. All authors engaged in data extraction and the performance of the research. AHP and $\mathrm{RD}$ participated in data analysis. All authors critically revised the manuscript, read and approved the final of the version to be published.

\section{References}

1. Hattori Y, Hattori K, Suzuki T, Matsuda N. Recent advances in the pathophysiology and molecular basis of sepsis-associated organ dysfunction: Novel therapeutic implications and challenges. Pharmacol Ther. 2017; 177: 56-66.

2. Hartman ME, Linde-Zwirble WT, Angus DC, Watson RS. Trends in the epidemiology of pediatric severe sepsis. Pediatr Crit Care Med. 2013; 14: 686-93.

3. Vincent J, Opal S, Marshall J, Tracey K. Sepsis definitions: time for change. Lancet. 2013; 381: 774-5.

4. Singer M, Deutschman CS, Seymour CW, Shankar-Hari M, Annane $\mathrm{D}$, Bauer M, et al. The Third International Consensus Definitions for Sepsis and Septic Shock (Sepsis-3). JAMA. 2016; 315: 801-10.

5. Marik P, Taeb A. SIRS, qSOFA and new sepsis definition. J Thorac Dis. 2017; 9: 943-5.

6. Lubis M, Lubis A, Nasution B. The usefulness of C-reactive protein, procalcitonin, and PELOD-2 score as a predictive factor of mortality in sepsis. Indones Biomed J. 2020; 12: 102-8.

7. Vincent JL. The clinical challenge of sepsis Identification and Monitoring. PloS Med. 2016; 13: e1002022. doi: 10.1371/journal. pmed.1002022.

8. Charles PE, Gibot S. Predicting outcome in patients with sepsis: new biomarkers for old expectations. Crit Care. 2014; 18: 108. doi: $10.1186 / \mathrm{cc} 13723$

9. Dauhan A, Lubis A, Lubis M. Vasoactive-inotropic score for early detection and mortality prediction of sepsis in children. Indones Biomed J. 2021; 13: 34-9.

10. Marshall JC, Vincent JL, Fink MP, Cook DJ, Rubenfeld G, Foster D, et al. Measures, markers and mediators: toward a staging system 
for clinical sepsis. A report of the fifth Toronto Sepsis Roundtable, Toronto, Ontario, Canada, October 25-26,2000. Crit Care Med. 2003; 31: 1560-7.

11. Kortgen A, Hoffmann G, Bauer M. Sepsis-current aspects of pathophysiology and implications for diagnosis and management. Eur J Trauma. 2006; 32: 3-9.

12. Lipinska-Gediga M. Neutrophils, NETs, NETosis - old or new factors in sepsis and septic shock? Anaethesiol Intens Ther. 2017; 49: 23540 .

13. Hamaguchi S, Hirose T, Akeda Y, Matsumoto N, Irisawa T, Seki M, et al. Identification of neutrophil extracellular traps in the blood of patients with systemic inflammatory response syndrome. J Int Med Res. 2013: 41: 162-8.

14. Wildhagen KC, Wiewel MA, Schultz MJ, Horn J, Schrijver R, Reutelingsperger $\mathrm{CP}$, et al. Extracellular histone $\mathrm{H} 3$ level are inversely correlated with antithrombin level and platelet counts and are associated with mortality in sepsis patients. Thromb Res. 2015; 136: 542-7.

15. Pan B, Alam HB, Chong W, Mobley J, Liu B, Deng Q, et al. Cit H3: a reliable blood marker for diagnosis and treatment of endotoxic shock. Sci Rep. 2017; 7: 8972. doi: 10.1038/s41598-017-09337-4.

16. Meng W, Paunel-Gorgulu A, Flohe S, Hoffmann A, Wittee I, MacKenzie C, et al. Depletion of neutrophil extracellular traps in vivo results in hypersusceptibility to polymicrobial sepsis in mice. Crit Care. 2012; 16: R137. doi: 10.1186/cc11442.

17. Hampson P, Dinsdale RJ, Pharmacol M, Wearn CM, Bamford AL, Bishop JRB, et al. Neutrophil dysfunction, immature granulocytes, and cell-free DNA are early biomarkers of sepsis in burn-injured patients. Ann Surg. 2017; 265: 1241-9.

18. Czaikoski PG, Mota JMSC, Nascimento DC, Sonego F, Castanheira FVS, Melo PH, et al. Neutrophil extracellular traps induce organ damage during experimental and clinical sepsis. Plos One. 2016; 11: e0148142. doi: 10.1371/journal.pone.0148142.

19. Thalin C, Daleskong M, Gorausson SP, Schafzberg D, Lasselin J, Laska A, et al. Validation of an enzyme-linked immunosorbent assay for the quantification of citrullinated histone $\mathrm{H} 3$ as a marker for neutrophil extracellular traps in human plasma. Immunol Res. 2017; 65: 706-12.

20. Hoppenbrouwers T, Boeddha NP, Ekinci E, Emonts M, Hazelhet JA, Driessen GJ, et al. Neutrophils extracellular traps in children with meningococcal sepsis. Pediatr Crit Care Med. 2018; 19: e286-91.
21. Viechtbauer W, Smits L, Kotz D, Bude L, Spigt M, Serroyen J, et al. A single formula for the calculation of sample size in pilot studies. J Clin Epidemiol. 2015; 68: 1375-9.

22. Goldstein B, Giroir B, Randolph A, the Member of the International Consensus Conference on Pediatric Sepsis. International pediatric sepsis consensus conference: Definitions for sepsis and organ dysfunction in pediatrics. Pediatr Crit Care Med. 2005; 6: 2-8.

23. Szima S, Balazs G, Elek N, Dahlem P. Pediatric sepsis: clinical markers. J Child Sci. 2017; 1: e42-53.

24. Tian Y, Russo RM, Li Y, Karmakar M, Liu B, Puskarich MA, et al. Serum citrullinated histone $\mathrm{H} 3$ concentrations differentiate patients with septic verses non-septic shock and correlate with disease severity. Infection. 2021; 49: 83-93.

25. Clark SR, Ma AC, Tavener SA, McDonald B, Goodarzi Z, Kelly MM, et al. Platelet TLR4 activates neutrophil extracellular traps to ensnare bacteria in septic blood. Nat Med. 2007; 13: 463-9.

26. McDonald B, Urrutia R, Yipp BG, Jenne CN, Kubes P. Intravascular neutrophil extracellular traps capture bacteria from the bloodstream during sepsis. Cell Host Microbe. 2012; 12: 324-33.

27. Li Y, Liu B, Fukudome EY, Lu J, Chong W, Jin G, et al. Identification of $\mathrm{Cit} \mathrm{H} 3$ as a potential serum protein biomarker in a lethal model of LPS-induced shock. Surgery. 2011; 150: 442-51.

28. Hirose T, Hamaguchi S, Matsumoto N, Irisawa T, Seki M, Tasaki O, et al. Presence of neutrophil extracellular traps and citrullinated histone $\mathrm{H} 3$ in the bloodstream of critically ill patients. PLoS One. 2014; 9: e111755. doi: 10.1371/journal.pone.0111755.

29. Bystrzycka W, Manda-Handzlik A, Sieczkowska S, Moskalik A, Demkow U, Ciepiela O. Azitromycin and chloramphenicol diminish neutrophil extracellular traps (NETs) release. Int J Mol Sci. 2017; 18: 2666. doi: 10.3390/ijms18122666.

30. Manda-Handzlik A, Bystrzycka W, Sieczkowska S, Demkow U, Ciepiela O. Antibiotics modulate the ability of neutrophils to release neutrophil extracellular traps. Adv Exp Med Biol. 2017; 944: 47-52

31. Bystrzycka W, Moskalik A, Sieczkowska S, Manda-Handzlik A, Demkow U, Ciepiela O. The effects of clindamycin and amoxicillin on neutrophil extracellular trap (NET) release. Cent Eur J Immunol. 2016; 41: 1-5. doi: 10.5114/ceji.2016.58811.

32. Li Y, Liu B, Fukudome EY, Lu J, Chong W, Jin G, et al. Identification of $\mathrm{Cit} \mathrm{H} 3$ as a potential serum protein biomarker in a lethal model of LPS-induced shock. Surgery. 2011; 150: 442-51. 